\title{
THE SATURNIAN 'GAS-DOUGHNUT’ HYPOTHESIS
}

\author{
THOMAS R. MCDONOUGH and NEIL BRICE
}

School of Electrical Engineering, Cornell University, Ithaca, N.Y., U.S.A.

\begin{abstract}
It is hypothesized that constituents escaping the atmosphere of Titan are unlikely to have sufficient velocity to escape from Saturn. Thus most of the material will enter into elliptical orbits which, at some point, cross Titan's orbit at a small angle. In total, this matter will form a 'doughnut' shaped feature, roughly centered on Titan's orbit and having dimensions of about $20 R_{\mathrm{S}}$ (Saturn radii) in the Saturnian-equatorial plane and $10 R_{\mathrm{S}}$ normal to this plane. It is assumed that the material is lost from the doughnut when it is ionized or is recaptured by Titan's atmosphere. The upper limit on the density of hydrogen in the ring is estimated to be about $10^{3} \mathrm{~cm}^{-3}$, with up to $99 \%$ of the particles in the doughnut being recaptured by Titan.

A brief summary of the work which has been done on this problem is given, and areas needing further research are suggested.
\end{abstract}

It has been calculated that most atoms or molecules which escape from the atmosphere of Titan probably do not have enough speed to escape from Saturn (McDonough and Brice, 1973a). If this is true, then the Titanian constituents will orbit Saturn, forming a gaseous ring around the planet which is much larger than the visible rings of Saturn. The purpose of this note is to summarize the current theoretical studies of this ring, which have been given by McDonough and Brice (1973a, b), McDonough (1974), Dennefeld (1973, 1974), Tabarié (1974), and Sullivan (1973), and to point out areas needing further research.

If the particles which escape Titan but not Saturn are unperturbed, they will describe elliptical orbits which intersect Titan's orbit. Because they intersect Titan's orbit, the interesting possibility exists that some of them will be recaptured by Titan. If Titan has an exospheric radius comparable to its visible radius, the fraction of particles which is recaptured is apt to be small, but if the exospheric radius is of the order of 10 Titan radii, the recaptured fraction could be as much as $~ 99 \%$ (McDonough and Brice, 1973a, b). Hunten (1974) has suggested that recapture may increase the gross escape rate from Titan in such a way that the net loss rate is the same as if there were no capture.

There are several potential sources of perturbations of the particle orbits, each of which may decrease the recapture probability: particle-particle collisions, solar radiation pressure, gravitational attraction by the satellites and by the aspherical component of the Saturnian gravitational field, interaction with the visible rings, interaction with the solar wind, and, if Saturn possesses a magnetic field, interaction with corotating magnetospheric plasma and energetic particles.

The importance of particle-particle collisions has been emphasized by Hunten (1974) and by Dennefeld (private communication), and has been studied under certain approximations by Sullivan (1973). The effect of such collisions is unclear and should be investigated. Solar radiation pressure, particularly that of the $L \alpha$ line of atomic hydrogen, may be significant despite the fact that the pressure is approximately 
balanced by the solar gravitational force, because (1) in Saturn's frame of reference, i.e., in free fall, the solar gravitational force is balanced (neglecting field gradients) by the centrifugal force due to motion around the Sun, while the atoms still experience radiation pressure, and (2) the quantization of the solar photons implies discontinuities in particle velocities. The principal effect of corotating plasma, if Saturn has a strong magnetic field, and of the solar wind, if it has not, is expected to be charge-exchange ionization of the orbiting atoms and molecules. Because the principal constituent of the doughnut is probably hydrogen, if Trafton's (1972) detection of Titanian $\mathrm{H}_{2}$ is correct, charge-exchange will probably control the ratio of atomic to molecular hydrogen in the doughnut (Blamont and Dennefeld, private communications). All of the above effects on particle orbits deserve further study.

For a Titanian exospheric temperature of $\sim 100 \mathrm{~K}$, celestial mechanics implies a hydrogen torus with a radius of the order of 20 Saturn's radii and with a thickness of the order of 10 Saturnian radii normal to Titan's orbital plane. Using the Titanian escape fluxes of Trafton (1972) and Hunten (1973), one can compute an upper limit to the hydrogen density in the doughnut of $\sim 10^{3} \mathrm{~cm}^{-3}$, if recapture by Titan is efficient (McDonough and Brice, 1973a, b). A smaller recapture efficiency could increase this density.

The Saturnian 'doughnut' hypothesis appears to be viable, and is worth pursuing theoretically and experimentally as a probe of the Saturn-Titan system.

\section{Acknowledgements}

We are indebted to Drs J. E. Blamont and M. Dennefeld of the University of Paris, and to Dr D. M. Hunten of Kitt Peak National Observatory, for stimulating conversations. This research was sponsored in part by the NASA Physics and Astronomy Program under Grant number 33-010-161, and the National Science Foundation Atmospheric Sciences Section under Grant numbers GA-11415 and GA-36916.

\section{References}

Dennefeld, M.: 1973, Ph. D. Thesis, University of Paris.

Dennefeld, M.,: 1974, this volume, p. 471.

Hunten, D. M.: 1973, J. Atmospheric Sci. 30, 726.

Hunten, D. M. (ed.): 1974, The Atmosphere of Titan (NASA SP-340), p. 110.

McDonough, T. R.: 1974, in D. M. Hunten (ed.), The Atmosphere of Titan (NASA SP-340), p. 118.

McDonough, T. R. and Brice, N. M.: 1973a, Nature 242, 513.

McDonough, T. R. and Brice, N. M.: 1973b, Icarus 20, 136.

Sullivan, R.: 1973, to be published.

Tabarié, N.: 1974, in D. M. Hunten (ed.), The Atmosphere of Titan (NASA SP-340), p. 123.

Trafton, L.: 1972, Astrophys. J. 175, 285.

\section{DISCUSSION}

Smith: Will the solar wind not tend to sweep out the proposed exospheric cloud of hydrogen?

McDonough: In deriving the density of the gaseous torus, we first assumed that charge-exchange of 
the hydrogen with solar wind was the dominant loss process. For $\mathrm{H}$, this lifetime is about $6 \mathrm{yr}$ at Saturn's distance, and for $\mathbf{H}_{2}$, about 50 yr. Thus the lifetimes are quite long, and you can get densities up to about $10^{5} \mathrm{~cm}^{-3}$ even with the solar wind unshielded by a Saturnian magnetosphere. Our second model included recapture by Titan. If there is recapture, the density could still be as much as about $10^{3} \mathrm{~cm}^{-3}$.

Baum: What observations would best set a limit on the amount of gas in the postulated 'Titan' ring? Can we already put a limit on it from existing data? Might spectra of Titan be detectably different when at different positions in its orbit and therefore seen through different amounts of the ring?

McDonough: L $\alpha$ and $\mathrm{H} \alpha$ (Balmer alpha) observations appear to be the most promising method of detecting or setting limits to the proposed Saturnian gas-torus. Existing $\mathrm{L} \alpha$ and $\mathrm{H} \alpha$ data do not appear to be sufficient to detect even the densest hydrogen torus. A previous search for an atmosphere around the visible rings of Saturn did not appear to set useful limits on the probable constituents of the Saturnian gas-torus. It is possible that Titanian spectra may be different at different positions, due to the torus, but the torus would be essentially transparent in most wavelengths, $L \alpha$ being a possible exception.

Gorgolewski: Is your model applicable to Jupiter satellites?

McDonough: Yes, it may apply to all of the giant planets. Jupiter's magnetosphere protects such a toroidal ring from the solar wind, although corotating plasma may reduce a ring's density somewhat. Two of Jupiter's Galilean satellites have been proposed as sites of atmospheres, and all of the Galilean satellites are deep enough in the gravitational potential well of Jupiter for them to trap escaped atmosphere in toroidal rings around Jupiter. 